\title{
Aging and Potential for Self-Renewal: Hydra Living in the Age of Aging - A Mini-Review
}

\author{
Ralf Schaible Meir Sussman Boris H. Kramer \\ Max Planck Institute for Demographic Research, Rostock, Germany
}

\section{Key Words}

Senescence · Aging · Trade-offs · Regeneration · Renewing · Nonsenescence $\cdot$ Maintenance

\begin{abstract}
Hydra present an interesting deviation from typical life histories: they have an extensive capacity to regenerate and self-renew and seem to defy the aging process. Hydra have the ability to decouple the aging process from their life history and therefore provide us with a unique opportunity to gain insight into the aging process not only for basal hydrozoans but also for other species across the tree of life. We argue that under steady feeding and asexual reproduction Hydra species are able to escape aging as a result of high levels of cell proliferation and regenerative ability. We further highlight cellular processes for stem cell maintenance, such as the telomere dynamic, which prevent the accumulation of damage and protect against diseases and pathogens that mediate this condition. In addition, we discuss the causes of aging in other Hydra species.

\section{Introduction}

While organisms across the tree of life display a broad range of life histories, only a few known species escape aging or show the ability to extend their lifespan in response to environmental signals. Aging and senescence are defined as the increasing probability of death with increasing age [1] (appendix 1). Aging studies conducted since the 1980s have demonstrated that mutated Caenorhabditis elegans individuals can live up to 5-6 times longer than wild-type controls [reviewed in 2]. Even in wild-type nematodes, the lifespan can be extended by switching into a 'dauer' larval stage, which enables larvae to halt development and arrest the aging process in order to escape starvation [3]. Such studies have sparked the hope that these life-extending mutations could be used to understand the underlying mechanisms that lead to senescence and to increase the lifespans of other organisms, such as human beings. They have also raised the questions of whether long-lived subpopulations could exist in nature and, if not, why the maximum lifespan is so rarely manifested in most aging organisms. Among several model organisms, a wide range of lifespans has been observed. Studies testing the fitness of long-lived $C$. elegans genotypes in competition with short-lived wild-

\section{KARGER}

E-Mail karger@karger.com

www.karger.com/ger
(C) 2014 S. Karger AG, Basel

0304-324X/14/0606-0548\$39.50/0
Ralf Schaible

Max Planck Institute for Demographic Research

Konrad-Zuse-Strasse 1

DE-18057 Rostock (Germany)

E-Mail schaible@ demogr.mpg.de 
Fig. 1. Nonsenescent and senescent-like pathways in Hydra [1, 5, 7]. Budding or asexual reproduction is the main reproductive output. Hydra can also reproduce sexually. After successful fertilization, the egg produces a theca and falls into the sediment. It then hatches and develops into a small Hydra - the new genet or clone lineage. All Hydra species escape senescence under benign conditions and while reproducing asexually. After the initiation of sexual reproduction, commonly triggered by, for example, environmental cues, 2 pathways are possible: in the nonsenescent pathway, individuals of some species $(H$. magnipapilla [1, 7] and $H$. vulgaris [5]) demonstrate neither a sexual or an asexual reproductive decline nor increased mortality. In contrast, while committed to the senescent-like pathway, adults of some $\mathrm{Hy}$ dra species, such as H. oligactis [7], die following the onset of sexual reproduction, possibly because investments in maintenance (i.e. survival), renewal, and clonal reproduction are abruptly neglected.

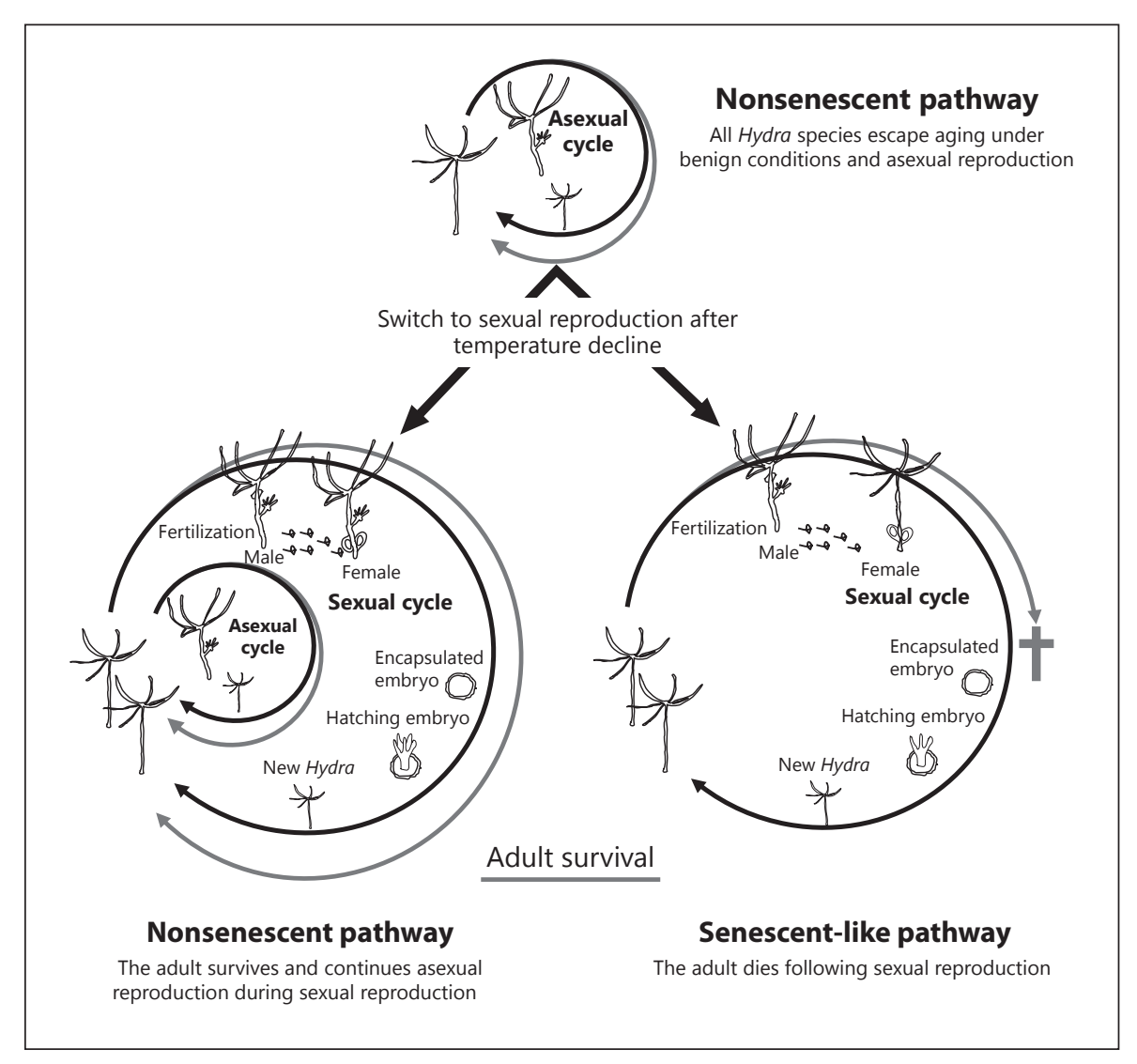

type worms have demonstrated that extension of the lifespan is not favored by natural selection, since longlived mutants have a lower level of fitness [4]. This may reflect the broad spectrum of lifespans found across the tree of life [1]. Meanwhile, human beings might be the only organisms that make concerted efforts to artificially increase their lifespan while postponing the aging process.

To understand the mechanisms that favor the evolution of extended lifespans and the biological causes of aging, the study of organisms that do not show senescence and live extremely long lives might be beneficial. Successful candidates for such studies would therefore be species that demonstrate both an increasing and a constant mortality pattern. Species of the genus Hydra meet this criterion (fig. 1).

Using Hydra as examples, this review will attempt to demonstrate that nonsenescence is not the result of selection towards a longer lifespan but is rather the consequence of mechanisms that evolved to increase the survival probability of individuals under harsh environmental conditions.

Aging and Potential for Self-Renewal: Hydra Living in the Age of Aging

\section{Closely Related Hydra Species Demonstrate Different Senescence Patterns}

\section{Nonsenescent Hydra}

In a pioneering study on Hydra vulgaris, a clonal Hydra species, Martinez [5] demonstrated a constant and low death rate with increasing age of individual polyps. For this purpose, 145 individuals were tracked under laboratory conditions for a period of over 4 years. Results from this study indicated that $H$. vulgaris individuals do not show aging, or that the process is so slow that aging was imperceptible over a period of 4 years. A recently published study confirmed these results and showed that, for H. magnipapillata strain 105, the risk of dying under laboratory conditions is low and does not change with age. This study estimated that under controlled conditions 5\% of the adults would still be alive after 1,400 years [1].

\section{Senescent-Like Hydra}

Brien [6] and Yoshida et al. [7] studied H. oligactis, a species which switches to a stage of sexual reproduction after a strong temperature decline (fig. 1). In the study of 
Yoshida et al. [7], both female and male cohorts showed an increase in mortality after becoming sexually active, with all individuals dying within 150 days at a low temperature.

Based on this experiment, it can be assumed that the life history of $H$. oligactis can be separated into 2 distinct stages that affect senescence patterns: a clonal reproductive period with high maintenance and low death rates, and a period of sexual reproduction in which senescence appears - a process which ultimately leads to the death of the polyps. The rapid death demonstrated by Yoshida et al. [7] may also resemble the life histories of semelparous organisms, in which sexual reproduction is triggered by declining temperatures or other environmental cues and is associated with an increase in mortality but not necessarily with progressive aging.

Other strains of $H$. oligactis might be cold adapted [see references in 8], and the closely related species $H$. magnipapilla [7] and H. vulgaris [5] do not demonstrate mortality either after the onset of sexual reproduction leading to the production of gametes and offspring or after temperature declines in which they keep producing new buds and show no signs of death or aging [5] (fig. 1).

\section{Nonaging under High Levels of Extrinsic Mortality Exists in Hydra and Challenges the Evolutionary Theories of Aging}

Our knowledge of Hydra survival and reproduction under natural environmental conditions is scant. A few studies have shown that, in addition to seasonal environmental factors, like temperature or water levels, biotic interactions with parasites, interspecies competition for resources, or high levels of predation can lead to considerable seasonal fluctuations in Hydra abundance [9-11]. Assuming high levels of extrinsic mortality risk, most individual Hydra die at an age that represents only a small fraction of their potential maximum lifespan. For these, the evolutionary advantage of a long life and nonsenescence seems to be irrelevant. A potentially unlimited lifespan that evolved under high levels of extrinsic mortality seems to contradict some aspects of the evolutionary theories of aging, i.e. that high levels of extrinsic mortality lead to the evolution of accelerated aging and a shorter intrinsic lifespan caused by a weak selection against lifeshortening mutations that do not compromise fitness [12].

Some theories attempt to bridge this paradox by linking novel adaptations to a longer lifespan (but without changes in aging patterns). For instance, some organisms' extended longevity can be associated with adaptations that reduce the risk of death due to environmental hazards (e.g. subterranean living [13] and group or social living [14]). Furthermore, preservation of seeds in plants, diapause and dauer formation (e.g. C. elegans) [3] are all excellent examples of the ability of organisms to halt their deterioration process or survive in unfavorable environmental conditions [15]. However, while many organisms extend their lifespan through various forms of dormancy, nonaging Hydra species have preserved (or maybe developed) a mechanism that enables constant lifespan extension in an environment without extrinsic mortality and without the need for hibernation or distinct phases of regeneration. We argue that the continuous stem cell proliferation and regenerative abilities of $\mathrm{Hydra}$ (fig. 2) have the following advantages, depending on the level of extrinsic mortality risk:

(i) Hydra are able to survive very harsh but nonlethal environmental conditions. With their profound cell proliferation capacity, Hydra are able to regenerate a whole organism even after being partly consumed by a predator or after being demolished by physical forces such as currents and storms. Moreover, Hydra species are extremely successful and establish populations in small freshwater reservoirs across a broad range of climates [16]. One important question guiding the study of the phenomenon of the nonaging of Hydra is whether their capacity for continuous proliferation has aided the dispersal of the species into highly competitive (mostly endemic) environments.

(ii) In an environment without extrinsic hazards, their extreme potential for renewal allows Hydra to escape senescence [5]. Here the question arises of why this extreme capacity for renewal is so rarely manifested in other organisms [for a review, see 17].

\section{High Levels of Cell Renewal and Cell Turnover Rates May Explain Why Some Hydra Strains Show No Senescence}

Organisms with a high renewal capacity are widely distributed across the tree of life, and these regeneration abilities are not restricted to basal or simple metazoans [18]. Clonal planarians [19] or basal flatworms like Macrostomum [20] demonstrate a similar regeneration by renewal, and it has been speculated that these organisms are long-lived and defy aging. In planarians and C. elegans worms, however, the functional link between their regeneration abilities and their extended lifespan is apparent. 


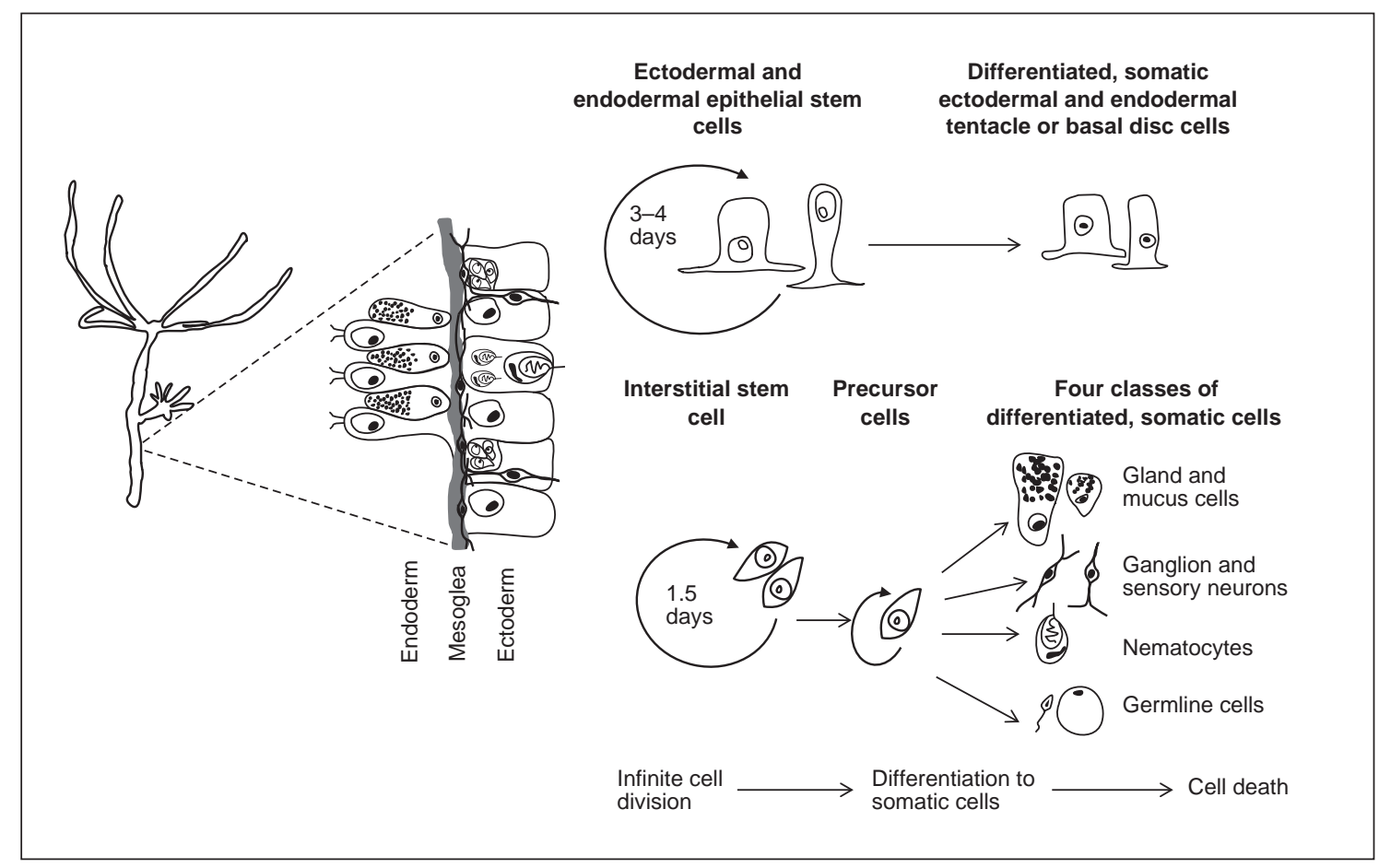

Fig. 2. Hydra body plan $[55,59]$. The freshwater polyp Hydra is a member of the phylum Cnidaria (Hydrozoa) and is characterized by a defined body plan consisting of stem cells, a simple nerve system, and 2 differentiated tissue layers (ectoderm and endoderm). Hydra consist of more than 3 active but separated stem cell communities: ectodermal epithelial stem cells, endodermal epithelial stem cells, and interstitial stem cell lineages. They are responsible for the extraordinary regeneration and survival ability of individual polyps. In Hydra, tissue comprised of all 3 stem cell lineages is highly dynamic, and all cells are continuously renewed every 20

These two groups have the same body plan, but planarian species have a regeneration capacity comparable to that of Hydra and can even regenerate a complete brain integrated with the preexisting nervous system [19]. Even when their cells deteriorate, cells can be replaced while organismal integrity is maintained, which means that these animals have no lifespan limits. In contrast, C. elegans shows low regeneration abilities and therefore has a limited lifespan [both reviewed in 18]. Moreover, in the more complex metazoans, the renewal potential declines over the course of development, and a high regenerative capacity is almost solely restricted to the embryo or juvenile stages [reviewed in 21]. Even primitive vertebrates like the zebrafish exhibit high levels of regenerative capacity throughout their life cycle [21]. In contrast to $\mathrm{Hy}$ $d r a$, clear evidence is lacking for a link between an extensive regeneration ability and nonaging. In humans and
30 days [see 59]. This continuous proliferation of epithelial and interstitial stem cells occurs in the central part of the gastric body from which cells migrate either to the upper or the lower body column or toward a growing bud. At both ends, unipotent epithelial stem cells differentiate into head and foot somatic tissue, thereby losing the potential for infinite cell division. Multipotent interstitial stem cells differentiate not only into nondividing somatic cells (e.g. nematocysts and nerve cells) but also into gametes, i.e the eggs and sperm. other vertebrates, aging seems to be inevitable. With increasing age, the regenerative capacity of organs and tissues is reduced. These structures are maintained rather than renewed in order to sustain the organismal integrity of these complex cellular and tissue structures. These examples demonstrate that, in organisms with an extraordinary potential for renewal and regeneration, physiological deterioration is reduced, which in turn reduces senescence. This mechanism is one strategy for extending the lifespan, and it might be a prerequisite for escaping senescence. Seen this light, a life-extending mechanism (e.g. continuous renewal of cells and tissues) must not be associated with a high cost, and it may be uncoupled from other processes or life history traits [17]. This suggests that, in all Hydra known to date, lifespan extension by renewal and proliferation are constitutively active; thus, there is no trade-off between constant renewal and termi- 
nal growth, asexual reproduction, and most maintenance tasks. Further, in many known Hydra species a detectable trade-off between constitutive renewal and sexual reproduction is also lacking, suggesting that these genetic pathways may have remained decoupled. However, in the senescent-like $H$. oligactis, the renewal of somatic cell lineages is dramatically affected by sexual reproduction [7], suggesting a possibly early trade-off between the pathways committed to extending life and those committed to sexual reproduction.

\section{Cell Renewal and Cell Turnover Rates in Relation to Various Life History Trade-Offs}

In general, aging patterns are assumed to be a result of various life history trade-offs. Life history strategies should be affected by the environmental conditions under which the species evolved. In this context, the trade-off between reproduction, growth, and survival is of particular interest and forms the basis for the 'disposable soma theory' [22]. As expected from life history theory, a change in one phenotypic trait like lifespan may be coupled with a change in another trait, because energy that is allocated to maintenance, which increases survival, cannot be used for other processes, such as reproduction. For the evolution of aging and nonaging, respectively, the trade-off or pleiotrophic effects between reproduction, somatic maintenance, and damage repair are of special interest.

In a single polyp, traits like size (number of cells per polyp), reproduction, and maintenance can be distinguished. The dynamic processes of cell loss and cell gain mediated by autophagy [23], apoptosis [24], continuous self-renewal, and differentiation are fundamental for the morphogenesis, reproduction, and maintenance of single polyps [25]. In the nonsenescent $H$. vulgaris, optimal life history trade-offs result in the continuous self-renewal of stem cells and tissues without any cost or disadvantage in gaining other traits [5]. This means that continuous selfrenewal simultaneously leads to nonsenescence, body growth, regeneration following injury, sexual reproduction, budding and the gathering of food by predation.

Schaible et al. [26] investigated how trade-offs between reproduction and maintenance (measured as survival under starvation) are modified under different environmental stressors. Under recurrent hormetic food stress, individuals emerge stronger in terms of health and reproductive activity, without degradation of one of their life history traits; hence, no trade-off between clonal reproduction and maintenance is apparent.
The opposite was observed in H. oligactis [7]: the shift from budding to sexual reproduction, which is triggered by a shift to lower incubating temperatures, leads to increased mortality. Such an extreme life history strategy is likely to evolve in small water bodies which desiccate in summer or freeze in winter, which makes the death of individuals inevitable $[7,9,10]$. Under such circumstances, a shift in allocation strategy from maintenance to reproduction could lead to the observed increase in mortality after sexual reproduction. When switching to sexual reproduction, $H$. oligactis produces robust eggs that can survive adverse conditions. Such channeling of resources towards sexual reproduction may result in postreproductive mortality, as was observed in the experiments of Yoshida et al. [7].

However, constant cell proliferation and high cell turnover rates in Hydra promote longevity and prevent deterioration that would lead to senescence. Therefore, the question arises of how Hydra maintain these constant cell renewal and turnover rates without any sign that they do so at the cost of other processes which might trigger aging. To explore this issue, we will take a closer look at the regulatory and cellular processes involved in the maintenance of the constant regeneration capability of Hydra throughout life. In particular, this includes the processes responsible for telomere maintenance, for protecting the individual from the accumulation of damages or pathogens/diseases, and for the regulation of the genetic operating system.

\section{Maintenance of High Cell Renewal and Turnover Rates}

\section{The Telomere Dynamic in Hydra}

Telomeres protect chromosomes against degradation, recombination, or random fusion by cellular DNA repair systems. Cellular senescence or cellular apoptosis starts if the telomere length falls below a certain threshold (the so-called Hayflick limit) [27]. The presence of the enzyme telomerase reverse transcriptase (TERT) can stop the telomere shortening that results from cell division by elongating the repetitive sequences within the telomere, which theoretically leads to an unlimited replicative capacity. On the other hand, continuous telomere-shortening might limit the ability to repair damaged cells, which, according to the telomere theory of aging, is believed to be the main reason why organisms are debilitated in later age and finally show an age-related increase in mortality. However, in the nonaging Hydra species, telomere dy- 
namics remain poorly understood. The telomere structure represented by the repetitive sequence TTAGGG and the affected proteins are conserved in $2 \mathrm{Hydra}$ species $[28,29]$. Indirect proof of a possible telomerase activity in Hydra and a prerequisite for constant cell proliferation and regeneration has been deduced from the presence of a gene-encoding TERT, as identified in the genome of $H$. magnipapillata [30]. Other species characterized by a high level of cell proliferation, such as sponges, preserve telomere integrity via a high telomerase activity [31]. In the planarian Schmidtea mediterranea, only the asexual organisms avoid senescence by maintaining a telomere length through telomerase activity, whereas the sexually reproducing organisms have to go through sexual reproduction to prevent telomere shortening [32]. Many invertebrates, fish, amphibians, and reptiles that show continuous cell proliferation to maintain a high regeneration ability require high telomerase activities [33]. In contrast, Traut et al. [28] described species of the class Calcarea (sponges), the phylum Cnidaria (sea anemones and jellyfish), and the phylum Placozoa which protect their telomeres against attrition with little or no telomerase activity. These observations suggest that the clonal lineages of Hydra, with a stable cell proliferation rate, might preserve their long-term telomere integrity via telomerase activity. However, there is no data available on how Hydra species inhibit telomere attrition.

\section{Protection against Damages}

Organisms continuously generate reactive oxygen species (ROS) as by-products of essential metabolic processes like the mitochondrial electron transport chain. If they are not intercepted by antioxidant defense, ROS induce oxidative cellular and molecular damages (proteins, lipids, mitochondria, and nuclear DNA). These damages are not only an important factor in the long-term fitness of clonal organisms, as they might be transferred unhindered to the next generations of individuals, but they can also influence fitness-related traits when damage occurs. In a final step, the age-related accumulation of ROS-induced damage might result in accelerated aging [34].

Therefore, long-lived Hydra individuals, at least in the laboratory, might be excellent model organisms for understanding the balance between free radical production and the antioxidant defense activity involved in preventing organismal deterioration. Many organisms, including Hydra, are protected against oxidative stress by antioxidant enzymes such as superoxide dismutase [35], glutathione peroxidases [36], and catalases [37]. Hydra individuals are able to regulate the expression of the mRNAs of all 3 enzymes, which indicates an active defense of antioxidants against ROS. However, the role of antioxidant defense in Hydra needs to be further studied.

Antioxidants act as a first line of defense against the accumulation of oxidative damage. An alternative way to eliminate oxidative damage to organelles or cells [38] and resynthesize damaged proteins and lipids is autophagy via lysosomes. Autophagy has therefore been identified as a key strategy for controlling damage levels [34]. In $\mathrm{Hy}$ $d r a$, autophagic vacuoles in epithelial cells can be frequently observed under starvation [23,39]. As the contents of these vacuoles provide food resources, autophagy has been identified as one of the main survival strategies of cells and organisms [25]. If such autophagic phases repeatedly occur in times of little or no food, Hydra individuals can constantly repair and renew their cellular biochemical arsenal by removing oxidized intermediates and through the elimination of damaged cells and mitochondria. The beneficial effects of maintenance and reproduction after starvation, demonstrated in the study of Schaible et al. [26], might be the consequence of an increased activation of autophagy followed by a rejuvenation effect for the remaining cells. This process appears to confer longterm benefits, as repeated induced autophagy phases may be assumed to prevent the accumulation of damage, which in turn reduces the aging effects [25].

\section{Protection against Diseases and Pathogens}

The immune system is a powerful mechanism for protecting an organism against environmental stress and pathogens. The main advantage of the immune system is that it is involved in creating good health, which can increase the ability of an organism to survive in a hazardous environment. But high costs associated with maintaining an efficient immune system may also be a disadvantage. As a result, maintenance of the immune system reduces the resources available for other life history traits, such as reproduction or other maintenance [40].

Hydra have a relatively simple, rapid, and nonspecific innate immune system [41] that is predominantly localized in the endoderm. In their immune defense, several cellular processes, like apoptosis, phagocytosis, and the production of antimicrobial peptides, might be involved. Rahat and Dimentman [42] showed that bacteria might be important for tissue proliferation and successful budding in Hydra; therefore, the innate immune system in Hydra needs to distinguish between beneficial symbionts and pathogens. This indicates that the innate immune system shapes the interaction between Hydra and microbiota [43]. Furthermore, they observed the same species- 
specific bacterial community between individuals captured in a natural pond and individuals that had been kept in laboratory conditions for 30 years [44]. These results are intriguing for researchers studying aging because, even among humans, an age-related change in gut microbiota can be observed [45]. The constant balance and long-term maintenance of Hydra with their associated bacterial community might indicate that $H y d r a$ have a well-preserved immune system without any age-related deterioration. It seems that the metabolic costs of maintenance of an innate immune defense are relatively low [46]. As the innate immune defense of Hydra is manifested at a low cost, its maintenance is not traded off against survival and reproduction. Consequently, the low cost of innate immunity and the long-term maintenance of bacteria-Hydra interactions might be a prerequisite for the nonaging pattern in Hydra. To prove age-specific deterioration of the innate immune system in Hydra, repeat measures of known-age individuals across a sufficient time span is necessary, but these are difficult to obtain because of the long lifespan of individual Hydra polyps under laboratory conditions.

The Genetic Operating System of Cell Renewal and the Cell Turnover Rate in Hydra

The gene regulation theory of aging proposes that senescence is the result of changes in gene expression [47]. Although it is clear that many genes show age-specific changes in expression, it is unlikely that selection could act on genes that promote senescence directly [47].

Studies of age-related genes and genetic pathways across distant animal phyla are important, as this type of research will help us to determine whether the identified principles are 'public or private', i.e. shared between distant related phyla (public) or only specific to distinct evolutionary lineages [48]. For instance, recent comparative studies have shown that some molecular determinants of lifespan, like the insulin pathway, appear to be conserved in different organisms across the tree of life (e.g. C. elegans, Drosophila melanogaster, and mice [48] and also Hydra [49]).

As noted above, Hydra have enormously effective and rapidly acting mechanisms for regenerating damaged body parts, which is a prerequisite for prolonged somatic maintenance [50] and which can lead to an extended life span without senescence [5]. Consequently, genes that have a direct effect on the aging mechanisms in Hydra should control stem cell behavior, their constant proliferation, their migration, and their differentiation potential.
The identification of the proximate genetic factors that are key to understanding Hydra longevity has already begun. Bridge et al. [51] identified FOXO, a transcription factor gene shown to control longevity [52], in the interstitial stem cell lineage and demonstrated its role in the stress response and in interstitial stem cell maintenance. Another study showed that FOXO might be involved in inducing the apoptosis of Hydra epithelial stem cells, a conserved function which may play a key role in maintaining body proportions [53]. Boehm et al. [54] detected a clear relationship between FOXO expression and stem cell homeostasis and maintenance, which influence stem cell proliferation and differentiation rates (from stem cells to somatic cells like head or foot cells). Besides FOXO, other genes like Oct4, a class5 POU domain protein, and the transcription factors of the $M y c$ family [both reviewed in 55], may play a significant role in controlling the dynamics of stem cell proliferation and are therefore suspected to play a major role in the longevity pattern of Hydra.

Whether these genes and their related mechanisms influence the lifespan or help to postpone aging is an interesting subject for future research.

\section{Conclusions}

In closely related $\mathrm{Hydra}$ species, we find alternative life histories and different patterns of senescence, which may have evolved as ecological adaptations.

Of particular importance is that in $H$. vulgaris and in other Hydra species the mechanisms for an extended lifespan and nonsenescence observed in the laboratory i.e. continuous cell proliferation and renewal - are also responsible for other pathways and traits, such as sexual reproduction, growth, and maintenance.

Without extrinsic mortality hazards, the constant cell proliferation and high cell turnover rates seem to be key mechanisms promoting longevity and preventing physiological decline, which in other organisms may lead to senescence. Furthermore, if a Hydra polyp is not exposed to extrinsic hazards, this mechanism allows it to maximize its maintenance level, which leads to nonsenescence. The costs of telomere maintenance, damage repair, and immune system maintenance do not seem to be linked, as they are not traded off against lifespan and reproduction. In contradiction to the disposable soma theory, this scenario suggests that lifespan extensions occur at no cost in Hydra [17]. 
Meanwhile, in $\mathrm{H}$. oligactis, a senescent relative of $\mathrm{Hy}$ $d r a$, the pathways promoting maintenance that lead to an extended lifespan and sexual reproduction seem to be coupled. Following environmental deterioration, which suggests inevitable death, individuals of $H$. oligactis start to reproduce sexually. They die, possibly because investments in maintenance (i.e. survival), renewal, and clonal reproduction are abruptly neglected. It would therefore be important for future research on aging in the Hydra genus to investigate whether the death of $H$. oligactis individuals is a consequence of intrinsic aging processes, which manifests the trade-off between reproduction and maintenance. Alternatively, specific $H$. oligactis strains exist which show different mortality or senescent-like scenarios respective of their ecological habitats.

The fact that Hydra may switch maintenance 'on' and 'off', leading to constant or increasing patterns of mortality, supports the claim that high levels of maintenance may be achieved in almost all organisms by molecular or physiological uncoupling. This assumption that organisms like Hydra are capable of switching aging on and off needs further validation. However, high levels of extrinsic mortality would lead to complicated experimental setups and the study of Hydra mutagenic strains may overcome this problem. In some organisms, individuals sharing identical genomes display a vast divergence of lifespans: (i) in the case of social hymenoptera, long-lived queens and short-lived workers can be raised from the same genome [56]; (ii) in the jellyfish Cassiopea spp. (Cnidaria: Scyphozoa), the medusa is short-lived while the polyp is long-lived [57], and (iii) in the hydrozoan Clytia hemisphaerica, the same individual metamorphoses from a long-lived to a short-lived stage [58].
As a member of the cnidarian phylum, situated at the base of metazoan life, Hydra present a remarkable life history that is bound to contribute to the future research on aging, lifespan extension, and cures for age-related ailments.

\section{Appendix}

Definition of demographic senescence [1]

A distinctive increase in the probability of death at older ages characterizes the classical senescence pattern observed for most mammals and bird species. The rise in mortality at older ages is caused by a progressive loss of vitality and functionality of the cells and tissues that form the organism. Apart from the predominantly increasing patterns of mortality, age-specific mortality may also be constant or declining. These patterns have been termed non-, negligible and negative senescence. Negative senescence patterns are found in some reptiles (tortoise), fish, and plants. Examples of nonsenescent species are mole rats, rockfish, and plants. However, the distribution of mortality patterns across the tree of life is highly diverse and appears to be random, as closely related species, such as different Hydra strains, may fall into distinct categories.

\section{Acknowledgements}

We would like to thank Janek Pilzecker for his help creating the figures. We thank the editor and two referees for their insightful and constructive comments and suggestions. This project was funded by the Max Planck Institute for Demographic Research in Rostock, Germany.

\section{References}

-1 Jones OR, Scheuerlein A, Salguero-Gómez R, Giovanni Camarda C, Schaible R, Brenda C, Dahlgren JP, Ehrlén J, García MG, Menges E, Quintana-Ascencio PF, Caswell H, Baudisch A, Vaupel JW: Diversity of ageing across the tree of life. Nature 2014;505:169-173.

$\checkmark 2$ Gems D, Partridge L: Genetics of longevity in model organisms: debates and paradigm shifts; in Julius D (ed): Annual Review of Physiology. Palo Alto, Annual Reviews, 2013, vol 75, pp 621-644.

3 Klass M, Hirsh D: Non-aging developmental variant of Caenorhabditis elegans. Nature 1976;260:523-525.
4 Chen J, Senturk D, Wang JL, Müller HG, Carey JR, Caswell H, Caswell-Chen EP: A demographic analysis of the fitness cost of extended longevity in Caenorhabditis elegans. Gerontol A Biol Sci Med Sci 2007;62:126-135.

-5 Martinez DE: Mortality patterns suggest lack of senescence in Hydra. Exp Geront 1998;33: 217-225.

6 Brien P: La perennite somatique. Biol Rev Camb Philos Soc 1953;28:308-349.

7 Yoshida K, Fujisawa T, Hwang JS, Ikeo K, Gojobori T: Degeneration after sexual differentiation in Hydra and its relevance to the evolution of aging. Gene 2006;385:64-70.

$\checkmark 8$ Martinez DE, Bridge D: Hydra, the everlasting embryo, confronts aging. Int J Dev Biol 2012;56:479-487.
-9 Bell G, Wolfe LM: Sexual and asexual reproduction in a natural population of Hydra pseudoligactis. Can J Zool 1985;63:851-856.

10 Cuker BE, Mozley SC: Summer population fluctuations, feeding, and growth of Hydra in an arctic lake. Limnol Oceanogr 1981;26:697708 .

11 Ribi G, Tardent R, Tardent P, Scascighini C: Dynamics of Hydra populations in Lake $\mathrm{Zu}$ rich, Switzerland, and Lake Maggiore, Italy. Schweiz Z Hydrol 1985;47:45-56.

12 Medawar PB: An unsolved problem of biology: an inaugural lecture delivered at University College, London, 6 December, 1951. London, Lewis, 1952.
Aging and Potential for Self-Renewal: Hydra Living in the Age of Aging 
13 Buffenstein R: The naked mole-rat? A new long-living model for human aging research. J Gerontol A Biol Sci Med Sci 2005;60:13691377.

14 Carey JR: Demographic mechanisms for the evolution of long life in social insects. Exp Gerontol 2001;36:713-722.

15 Lubzens E, Cerda J, Clark MS: Dormancy and resistance in harsh environments introduction; in Lubzens E, Cerda J, Clark M (eds): Dormancy and Resistance in Harsh Environments. Berlin, Springer, 2010, vol 21, pp 1-4.

16 Martinez DE, Iniguez AR, Percell KM, Willner JB, Signorovitch J, Campbell RD: Phylogeny and biogeography of Hydra (Cnidaria: Hydridae) using mitochondrial and nuclear DNA sequences. Mol Phylogenet Evol 2010; 57:403-410.

17 Schaible R, Sussmann M: FOXO in aging: did evolutionary diversification of FOXO function distract it from prolonging life? Bioessays 2013;35:1101-1110.

18 Agata K, Inoue T: Survey of the differences between regenerative and non-regenerative animals. Dev Growth Differ 2012;54:143-152.

$\checkmark 19$ Umesono Y, Agata K: Evolution and regeneration of the planarian central nervous system. Dev Growth Differ 2009;51:185-195.

20 Egger B, Ladurner P, Nimeth K, Gschwentner $\mathrm{R}$, Rieger R: The regeneration capacity of the flatworm Macrostomum lignano - on repeated regeneration, rejuvenation, and the minimal size needed for regeneration. Dev Genes Evol 2006;216:565-577.

-21 Seifert AW, Voss SR: Revisiting the relationship between regenerative ability and aging. BMC Biol 2013;11:2.

22 Kirkwood TBL: Evolution of aging. Nature 1977;270:301-304.

23 Chera S, Buzgariu W, Ghila L, Galliot B: Autophagy in Hydra: a response to starvation and stress in early animal evolution. Biochym Biophys Acta 2009;1793:1432-1443.

24 Boettger A, Alexandrova O: Programmed cell death in Hydra. Semin Cancer Biol 2007;17: 134-146.

25 Galliot B, Ghila L: Cell plasticity in homeostasis and regeneration. Mol Reprod Dev 2010; 77:837-855

26 Schaible R, Ringelhan F, Kramer BH, Miethe $\mathrm{T}$ : Environmental challenges improve resource utilization for asexual reproduction and maintenance in Hydra. Exp Gerontol 2011;46:794-802.

$\checkmark 27$ Hayflick L: Limited in vitro lifetime of human diploid cell strains. Exp Cell Res 1965;37:614636.

-28 Traut W, Szczepanowski M, Vitkova M, Opitz C, Marec F, Zrzavy J: The telomere repeat motif of basal metazoa. Chromosome Res 2007; $15: 371-382$
29 Anokhin B, Hemmrich-Stanisak G, Bosch TCG: Karyotyping and single-gene detection using fluorescence in situ hybridization on chromosomes of Hydra magnipapillata (Cnidaria: Hydrozoa). Comp Cyto 2010;4:97-110.

30 Steele RE, David CN, Technau U: A genomic view of 500 million years of cnidarian evolution. Trends Genet 2011;27:7-13.

31 Koziol C, Borojevic R, Steffen R, Muller WEG: Sponges (Porifera) model systems to study the shift from immortal to senescent somatic cells: the telomerase activity in somatic cells. Mech Ageing Dev 1998;100:107-120.

32 Tan TCJ, Rahman R, Jaber-Hijazi F, Felix DA, Chen C, Louis EJ, Aboobaker A: Telomere maintenance and telomerase activity are differentially regulated in asexual and sexual worms. Proc Natl Acad Sci USA 2012;109: 4209-4214.

33 Gomes NMV, Shay JW, Wright WE: Telomeres and telomerase; in Wolf NS (ed): Comparative Biology of Aging. New York, Springer, 2010, pp 227-258.

34 Kirkwood TBL, Kowald A: The free-radical theory of ageing - older, wiser and still alive: modelling positional effects of the primary targets of ROS reveals new support. Bioessays 2012;34:692-700.

35 Dash B, Metz R, Huebner HJ, Porter W, Phillips TD: Molecular characterization of two superoxide dismutases from Hydra vulgaris. Gene 2007;387:93-108.

-36 Dash B, Metz R, Huebner HJ, Porter W, Phillips TD: Molecular characterization of phospholipid hydroperoxide glutathione peroxidases from Hydra vulgaris. Gene 2006;381: $1-12$.

37 Dash B, Phillips TD: Molecular characterization of a catalase from Hydra vulgaris. Gene 2012;501:144-152.

38 Melendez A, Neufeld TP: The cell biology of autophagy in metazoans: a developing story. Development 2008;135:2347-2360.

-39 Bosch TCG, David CN: Growth-regulation in Hydra - relationship between epithelial-cell cycle length and growth-rate. Dev Biol 1984; 104:161-171.

40 Previtali MA, Ostfeld RS, Keesing F, Jolles AE, Hanselmann R, Martin LB: Relationship between pace of life and immune responses in wild rodents. Oikos 2012;121:1483-1492.

41 Miller DJ, Hemmrich G, Ball EE, Hayward DC, Khalturin K, Funayama N, Agata K, Bosch TCG: The innate immune repertoire in Cnidaria - ancestral complexity and stochastic gene loss. Genome Biol 2007;8:R59.

42 Rahat M, Dimentman C: Cultivation of bacteria-free Hydra viridis - missing budding factor in non-symbiotic Hydra. Science 1982; 216:67-68.
43 Bosch TCG: Understanding complex hostmicrobe interactions in Hydra. Gut Microbes 2012;3:345-351.

44 Fraune S, Bosch TCG: Long-term maintenance of species-specific bacterial microbiota in the basal metazoan Hydra. Proc Natl Acad Sci USA 2007;104:13146-13151.

$\checkmark 45$ Biagi E, Candela M, Turroni S, Garagnani P, Franceschi C, Brigidi P: Ageing and gut microbes: perspectives for health maintenance and longevity. Pharmacol Res 2013;69:11-20.

46 Lee KA: Linking immune defenses and life history at the levels of the individual and the species. Integr Comp Biol 2006;46:10001015.

47 Weinert BT, Timiras PS: Theories of aging. J App Physiol 2003;95:1706-1716.

48 Partridge L, Gems D: Mechanisms of ageing: public or private? Nat Rev Genet 2002;3:165175

49 Steele RE, Lieu P, Mai NH, Shenk MA, Sarras MP: Response to insulin and the expression pattern of a gene encoding an insulin receptor homologue suggest a role for an insulin-like molecule in regulating growth and patterning in hydra. Dev Genes Evol 1996;206:247-259.

50 Kirkwood TBL, Austad SN: Why do we age? Nature 2000;408:233-238.

51 Bridge D, Theofiles AG, Holler RL, Marcinkevicius E, Steele RE, Martinez DE: FoxO and stress responses in the cnidarian Hydra vulgaris. PLoS One 2010;5:e11686.

52 Kenyon CJ: The genetics of ageing. Nature 2010;464:504-512.

53 Lasi M, David CN, Boettger A: Apoptosis in pre-bilaterians: Hydra as a model. Apoptosis 2010;15:269-278.

-54 Boehm A-M, Khalturin K, Anton-Erxleben F, Hemmrich G, Klostermeier UC, Lopez-Quintero JA, Oberg H-H, Puchert M, Rosenstiel P, Wittlieb J, Bosch TCG: FoxO is a critical regulator of stem cell maintenance in immortal hydra. Proc Natl Acad Sci USA 2012;109: 19697-19702.

55 Hobmayer B, Jenewein M, Eder D, Eder M-K, Glasauer S, Gufler S, Hartl M, Salvenmoser $\mathrm{W}$ : Stemness in Hydra - a current perspective. Int J Dev Biol 2012;56:509-517.

56 Kramer BH, Schaible R: Colony size explains the lifespan differences between queens and workers in eusocial hymenoptera. Biol J Linn Soc Lond 2013;109:710-724.

57 Ojimi MC, Isomura N, Hidaka M: Telomerase activity is not related to life history stage in the jellyfish Cassiopea sp. Comp Biochem Physiol A Mol Integr Physiol 2009;152:240244.

58 Houliston E, Momose T, Manuel M: Clytia hemisphaerica: a jellyfish cousin joins the laboratory. Trends Genet 2010;26:159-167.

-59 Steele RE: Developmental signaling in Hydra: what does it take to build a 'simple' animal? Dev Biol 2002;248:199-219. 